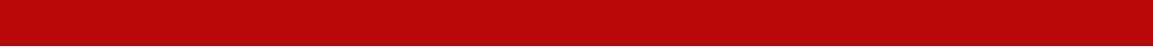

EDITORIALS

\title{
Diet, genes, and obesity
}

\author{
Genetic predisposition to obesity is no barrier to successful weight management
}

\author{
Louisa J Ells reader in public health and obesity ${ }^{1}$, Alessandro Demaio medical officer ${ }^{2}$, Nathalie \\ Farpour-Lambert program director ${ }^{3}$
}

${ }^{1}$ School of Health and Social Care, Teesside University, Middlesbrough, UK; ${ }^{2}$ Department of Nutrition for Health and Development, World Health Organization, Geneva, Switzerland; ${ }^{3}$ Obesity Prevention and Care Program Contrepoids, University Hospitals of Geneva, Geneva, Switzerland

Globally, the prevalence of obesity has tripled since 1975, with 671 million adults and 124 million young people (5-19 years) estimated to be affected in 2016. ${ }^{1}$ Given the serious associated health and economic consequences of obesity, ${ }^{2-4}$ finding effective weight management strategies is both a national and a global priority. ${ }^{56}$

Although behavioural interventions that improve dietary intake and increase physical activity can be effective in reducing body weight in adults, long term efficacy is often limited ${ }^{78}$ and it can be tempting to attribute failure to a genetic predisposition. Such discussions risk promoting a perception that policies and interventions to tackle obesity are futile, leading to loss of commitment and associated resources. Family, twin, and adoption studies show a moderate to high heritability for obesity, ${ }^{9}$ but monogenic causes of obesity are rare. Genetic predisposition in most people is polygenic. Important analyses of environment-gene interactions clearly show the detrimental effect of our obesogenic environment. ${ }^{910}$

The linked study by Wang and colleagues (doi:10.1136/bmj. j5644) examined the interaction between adherence to healthy dietary patterns and genetic predisposition to obesity in relation to long term weight loss. ${ }^{11}$ The authors assessed changes in body mass index and body weight between 1986 and 2006 in 8828 women and 5218 men from two large cohorts of US health professionals. Healthy dietary patterns were assessed using three dietary indices: the Alternate Healthy Eating Index 2010 (AHEI-2010), Dietary Approach to Stop Hypertension (DASH), and the Alternate Mediterranean Diet (AMED). The authors found that the association between a healthy diet and weight loss was stronger in participants with a greater genetic predisposition to obesity.

As with many genetic association studies, the effect of genetic predisposition was small. However, the findings provide encouraging new evidence that although a better diet can improve weight loss, the effect may be greatest in those with the highest genetic predisposition for obesity. The findings also complement previous studies undertaken by the study authors and others, ${ }^{12-14}$ showing that unhealthy products such as sugar sweetened drinks and fried food can magnify genetic associations with increased body mass index.

Both strands of evidence illustrate the importance of healthy dietary patterns in the prevention of weight gain, particularly in people at greatest risk. Wang and colleagues' findings further support the importance of national and international guidelines that define and promote adherence to healthy dietary patterns, ${ }^{1516}$ and help to dispel misconceptions that a genetic predisposition will inhibit successful weight management.

The authors acknowledge that the underlying biological mechanism driving the observed interaction remains unclear, and further research to characterise these mechanisms and explore the clinical implications of the findings would be helpful. They also acknowledge limitations including the influence of unmeasured confounders such as physical activity, the non-randomised adherence to healthy dietary patterns, and possible reverse causality. These considerations are important given the complex multifactorial nature of obesity. ${ }^{17}$ Furthermore, the results are generalisable only to other populations of health professionals of European descent.

The advantages of consuming a healthy diet are clear, but it is important to acknowledge the challenges of achieving this in an obesity promoting environment in which unhealthy food options are cheap, readily available in large portions, and heavily marketed. These findings reinforce the critical urgency of comprehensive policies that prioritise healthy food environments and systems, with an emphasis on vulnerable populations. These include fiscal policies and subsidies supporting healthy eating through pricing, improved product nutrition through reformulation, urban planning and zoning laws that ensure healthier food environments, childcare and school based food programmes that provide healthy food early in life, the protection of young people and families from the marketing of unhealthy products, trade and investment decisions that give healthy diets top priority, and closer congruence between government agricultural subsidies and national dietary recommendations. ${ }^{18-25}$ Finally, consumers must be empowered 
to make more informed choices through package labelling and portion size guidelines. ${ }^{26}$

In the newly announced United Nations Decade of Action on Nutrition, ${ }^{27}$ Wang and colleagues' study underlines the critical importance of achieving healthy diets for everyone. This is still a challenge for many, however ${ }^{28}$ with poor diet being a leading risk factor for death and disability globally. ${ }^{29}$ Genetic predisposition is no barrier to successful weight management and no excuse for weak health and policy responses. Through evidence based and cost effective interventions at both individual and societal levels, governments and populations must act to ensure universal healthy diets within health promoting food environments and food systems. This must become the new normal. Only then will we begin to curb and ultimately reverse the global epidemic of obesity.

Competing interests: We have read and understood the BMJ policy on declaration of interests and declare the following: $A D$ is a fulltime staff member for the World Health Organization; this article was written in his personal capacity, and its contents do not reflect the policies or views of any third party. LJE has a part time secondment to Public Health England but wrote this editorial in her role as Reader in public health and obesity at Teesside University.

Provenance and peer review: Commissioned; not peer reviewed.

1 NCD Risk Factor Collaboration (NCD-RisC). Worldwide trends in body-mass index underweight, overweight, and obesity from 1975 to 2016: a pooled analysis of 2416 population-based measurement studies in 128.9 million children, adolescents, and adults. Lancet2017;390:2627-42. doi:10.1016/S0140-6736(17)32129-329029897

2 Word Health Organization. Obesity and overweight. 2017. http://www.who.int/mediacentre/ factsheets/fs311/en/.

3 WangYCMcPhersonKMarshTGortmakerSLBrownM. Health and economic burden of the projected obesity trends in the USA and the UK. Lancet2011;378:815-25. doi:10.1016/S0140-6736(11)60814-321872750

4 WhitlockGLewingtonSSherlikerPProspective Studies Collaboration. Body-mass index and cause-specific mortality in 900000 adults: collaborative analyses of 57 prospective studies. Lancet2009;373:1083-96. doi:10.1016/S0140-6736(09)60318-419299006

5 Public Health England. From evidence into action: opportunities to protect and improve the nation's health.Public Health England, 2014.

$6 \quad$ World Health Organization. Global action plan for the prevention and control of non-communicable diseases 2013-2020.WHO, 2013.

7 AvenellABroomJBrownTJ. Systematic review of the long-term effects and economic consequences of treatments for obesity and implications for health improvement. Health Technol Assess2004;8:iii-iv, 1-182. doi:10.3310/hta821015147610
8 SimpsonSAShawCMcNamaraR. What is the most effective way to maintain weight loss in adults?BMJ2011;343:d8042. doi:10.1136/bmj.d804222205707

9 HinneyAVogelClHebebrandJ. From monogenic to polygenic obesity: recent advances. Eur Child Adolesc Psychiatry2010;19:297-310. doi:10.1007/s00787-010-0096-620127379

10 GoodarziMO. Genetics of obesity: what genetic association studies have taught us about the biology of obesity and its complications. Lancet Diabetes Endocrinol2017;S2213-8587(17)30200-0.28919064

11 WangTHeianzaYSunD. Improving adherence to healthy dietary patterns, genetic risk, and long term weight gain: gene-diet interaction analysis in two prospective cohort studies. BMJ2018;360:j5644.

12 QiQChuAYKangJH. Sugar-sweetened beverages and genetic risk of obesity. N Engl J Med2012;367:1387-96. doi:10.1056/NEJMoa120303922998338

13 QiQChuAYKangJH. Fried food consumption, genetic risk, and body mass index: gene-diet interaction analysis in three US cohort studies. BMJ2014;348:g1610. doi:10.1136/bmj.g161024646652

14 BrunkwallLChenYHindyG. Sugar-sweetened beverage consumption and genetic predisposition to obesity in 2 Swedish cohorts. Am J Clin Nutr2016;104:809-15. doi:10.3945/ajcn.115.12605227465381

15 Public Health England. Government recommendations for energy and nutrients for males and females aged $1-18$ years and 19+ years.Public Health England, 2016.

16 World Health Organization. Healthy diet. 2015. http://www.who.int/nutrition/publications/ nutrientrequirements/healthydiet_factsheet394.pdf.

17 ButlandBJebbSKopelmanP. FORESIGHT. Tackling obesities: future choices - project report.2nd ed. Government Office for Science, 2007.

18 World Health Organization. Dietary interventions for the appendix 3 of the global action plan for non communicable disease. 2017. www.who.int/ncds/governance/unhealthy_diet. pdf?ua $=1$.

19 TedstoneATargettAAllenR. Sugar reduction: from evidence to action. Public Health England, 2015.

20 Government HM. Childhood obesity: a plan for action. 2016. https://www.gov.uk/ government/publications/childhood-obesity-a-plan-for-action.

21 Public Health England. Sugar reduction and wider reformulation programme: interim review.PHE, 2017.

22 World Health Organization. Report of the commission on ending childhood obesity.WHO, 2016

23 World Health Organization. Set of recommendations on the marketing of foods and non-alcoholic beverages to children.WHO, 2010.

24 World Health Organization. Fiscal policies for diet and prevention of noncommunicable diseases. WHO, 2015.

25 RutterHCavillN. Obesity and the environment: regulating the growth of fast food outlet.Public Health England, 2014

26 CecchiniMWarinL. Impact of food labelling systems on food choices and eating behaviours: a systematic review and meta-analysis of randomized studies. Obes Rev2016;17:201-10. doi:10.1111/obr.1236426693944

27 World Health Organization. United Nations Decade of Action on Nutrition. 2017. http:// www.who.int/nutrition/decade-of-action/en/

28 BatesBLennoxAPrenticeA. NDNS: results from years 1 to 4 (combined).Public Health England, 2014.

29 GBD 2016 Risk Factors Collaborators. Global, regional, and national comparative risk assessment of 84 behavioural, environmental and occupational, and metabolic risks or clusters of risks, 1990-2016: a systematic analysis for the Global Burden of Disease Study 2016. Lancet2017;390:1345-422. doi:10.1016/S0140-6736(17)32366-828919119

Published by the BMJ Publishing Group Limited. For permission to use (where not already granted under a licence) please go to http://group.bmj.com/group/rights-licensing/ permissions 